\section{Preescolar masculino con Síndrome Prader-Willi y tromboembolia pulmonar}

\author{
Preschool child with Prader- \\ Willi Syndrome and pulmonary \\ thromboembolism
}

Miriam Padrón-Martínez ${ }^{1}$ Armando Partida-Gaytán ${ }^{2}$ Cecilia Ridaura-Sánz ${ }^{3}$

${ }^{1}$ Endocrinóloga Pediatra, adscrita a Clínica de Obesidad.

${ }^{2}$ Coeditor Acta Pediátrica de México.

${ }^{3}$ Patólogo, Jefe de Servicio de Estudios Post mortem. Instituto Nacional de Pediatría, Ciudad de México.

\section{RESUMEN CLÍNICO}

El paciente fue referido a los 20 días de vida por aumento de perímetro cefálico, separación de suturas, síndrome dismórfico y problemas en la alimentación con ahogamiento, cianosis, Ilanto débil y estridor laríngeo desde el nacimiento.

Dentro de los antecedentes relevantes tuvo una madre adolescente (16 años) que cursó con infección de vías urinarias y fiebre tifoidea durante el primer trimestre del embarazo, recibiendo tratamiento no especificado. Fue obtenido por vía abdominal por presentación pélvica y circular de cordón. Su somatometría al nacimiento fue normal. Su esquema de inmunizaciones se reportó incompleto con la aplicación de una dosis de Sabin únicamente. La exploración física inicial documentó hipoactividad, Ilanto débil, estridor laríngeo, perímetro cefálico en centil 50, fontanela anterior y posterior amplias, normotensas. Cara alargada, pabellones auriculares normales, boca en carpa con paladar alto, sin macroglosia. Tórax con teletelia, abdomen globoso sin hernias ni visceromegalias. Pene con prepucio estrecho, sin hipospadias. Escroto con bolsas vacías, testículos palpables en canal inguinal. Extremidades con pliegue simiesco en mano izquierda. Reflejos de succión, búsqueda y Moro positivos, hipotonía generalizada, reflejos miotáticos y clono positivos.

Ante los hallazgos neurológicos asociados con síndrome dismórfico se realizaron estudios de extensión. El ultrasonido transfontanelar identificó quiste de septo, secuelas de hemorragia ventricular sin dilatación ventricular. Perfil tiroideo, tamiz me-
Recibido: 13 de febrero del 2015

Aceptado: 17 de febrero del 2015

Correspondencia: Dra. Cecilia Ridaura Sánz Instituto Nacional de Pediatría. Insurgentes Sur 3700-C CP 04530 México, D.F.

Tel: 10840900 ext: 1123 cridaura@gmail.com

Este artículo debe citarse como Padrón-Martínez M, Partida-Gaytán A, Ridaura-Sánz C. Preescolar masculino con Síndrome PraderWilli y tromboembolia pulmonar. Acta Pediatr Mex 2015;36:105-113. 
tabólico ampliado, cariotipo, electromiografía y velocidad de conducción reportados como normales, descartando hipotiroidismo congénito, errores innatos del metabolismo más frecuentes, cromosomopatías y atrofia espinal atribuyendo hipotonía a causa central.

Se inició rehabilitación y seguimiento ambulatorio; presentó discreta mejoría en tono y fuerza muscular logrando sostén cefálico a los 8 meses y sedestación a los 17 meses. A los 18 meses se realizó tomografía axial computada que documentó atrofia cortical moderada en regiones frontoparietales y aplanamiento de circunvoluciones cerebrales en forma generalizada, evidenciando probable alteración en la migración celular.

A los 20 meses se integró diagnóstico de obesidad con peso de $14.3 \mathrm{~kg}$ (centiles 90-95), longitud de $86 \mathrm{~cm}$ (centiles 50-75) por lo que se solicitó evaluación por endocrinología pediátrica que documentó persistencia de criptorquidia, sin micropene. Se realizó prueba de reserva testicular con respuesta normal por lo que se recomendó orquidopexia y alimentación baja en hidratos de carbono simples.

El paciente perdió seguimiento y regresó a nuestra institución hasta los 3 años 11 meses al servicio de Urgencias Pediátricas por infección de vías aéreas superiores de una semana de evolución. No mejoró con el tratamiento ambulatorio y se agregaron, en las 48 horas previas: irritabilidad, rechazo a la vía oral, flacidez generalizada, dificultad respiratoria, somnolencia, falta de respuesta a estímulos verbales o dolorosos y fiebre. Al ingreso pesó 27 kg (centil > 97) midió 106 cm (centil 75) y en la exploración se encontró irritable, quejumbroso, con estridor laríngeo, hiperemia de faringe y amígdalas hipertróficas. Rudeza respiratoria en campos pulmonares. Abdomen globoso por panículo adiposo abundante, sin visceromegalias.
Pene pequeño, testículos palpables. Flacidez generalizada, disminución de fuerza muscular, rigidez de nuca dudosa, reflejos osteotendinosos disminuidos y Babinski bilateral.

Se le hospitalizó y se iniciaron estudios de extensión ante un proceso infeccioso con sintomatología respiratoria y neurológica. La radiografía de tórax mostró un patrón restrictivo y cardiomegalia. En una radiografía lateral del cuello se observó crecimiento adenoideo con obstrucción de $70 \%$ de la columna de aire. Se documentó virus sincitial respiratorio en panel respiratorio. El líquido cefalorraquídeo se reportó xantocrómico, sin película, con proteínas de $129 \mathrm{mg} / \mathrm{dL}$, glucosa $74 \mathrm{mg} / \mathrm{dL}$ y 3 células. Se ingresó y manejó con los diagnósticos de obesidad exógena, retraso psicomotor, síndrome hipotónico, probable síndrome Prader-Willi, amigdalitis, laringotraqueitis y probable neuroinfección viral. Evolucionó favorablemente con tratamiento hospitalario y fue egresado a los 10 días para continuar estudio por consulta externa.

A los 4 años se realizó hibridación in situ con fluorescencia (FISH) con la sonda de PraderWilli/Angelman confirmando 46XY 1SH del (15) (q11-q12) y se demostró deleción de la región crítica.

A los 4 años 7 meses cursó con infección de vías respiratorias superiores con dificultad respiratoria que requirió manejo hospitalario en otra institución. Dos meses después acudió a nuestra institución con infección de vías respiratorias superiores que no mejoró tras 2 semanas de tratamiento ambulatorio con antibióticos y nebulizaciones, agregándose fiebre y dificultad respiratoria. Ingresó con diagnóstico de neumonía adquirida en la comunidad grave con insuficiencia respiratoria. En las primeras horas presentó deterioro neurológico con crisis convulsiva tonicoclónica generalizada, cianosis y deterioro respiratorio; requirió apoyo mecánico a 
la ventilación. La radiografía de tórax documentó infiltrado bronconeumónico y cardiomegalia. La gasometría arterial inicial con alcalosis metabólica crónica e hipoxemia. A pesar de tratamiento integral la capacidad ventilatoria no mostró mejoría y el décimo día de internamiento presentó súbitamente cianosis central, palidez importante y broncoconstricción sin respuesta a broncodilatador. Se documentó en radiografía de tórax una imagen heterogénea radiopaca que abarcó todo el hemitórax derecho con borrado del ángulo costodiafragmático y se consideró neumonía complicada con derrame pleural. Se agregó sangrado por cánula, pulsos débiles e hipotensión. Se sospechó edema pulmonar, se restringieron líquidos, se reportó una presión venosa central de $10 \mathrm{cmH}_{2} \mathrm{O}$, se inició apoyo aminérgico pero no hubo mejoría presentando un primer paro cardiorrespiratorio de 15 minutos $y$, 5 horas después, un segundo paro cardiorrespiratorio irreversible.

\section{DISCUSIÓN CLÍNICA}

El síndrome de Prader-Willi es un trastorno genético raro, originalmente descrito en 1956 por Prader, Labhart y Willi. Su prevalencia es variable pero se estima entre 1:10 000-25 000 nacidos vivos. $^{1-3}$ La causa genética es la ausencia de la expresión de genes heredados del padre en la región cromosómica 15q11-q13, ya sea por deleción, disomía uniparental materna, defecto de impronta genética o translocación balanceada. ${ }^{2}$

El cuadro clínico se caracteriza por hipotonía neonatal, rasgos faciales atípicos, criptorquidia, hipogenitalismo inicialmente, posteriormente hipogonadismo, talla baja y discapacidad intelectual de grado variable. La hipotonía se asocia con hipomotilidad fetal y frecuentemente la presentación pélvica dificulta el nacimiento por parto. Con frecuencia los pacientes tienen alteraciones de succión y deglución que dificultan la alimentación y pueden provocar fallo de medro. En etapas posteriores de la vida otras características son evidentes: síndrome de apnea hipopnea obstructiva del sueño, alteraciones del comportamiento, trastornos psiquiátricos, hiperfagia, obesidad mórbida y sus complicaciones (i.e. diabetes mellitus, dislipidemias, enfermedad isquémica coronaria, entre otras). El síndrome de Prader-Willi es la causa genética más frecuente de obesidad grave. ${ }^{2}$

En el año que falleció este paciente (1998) el tratamiento del síndrome de Prader-Willi consistía en intervenciones dietéticas, ambientales y del estilo de vida, así como rehabilitación física y neurológica con la finalidad de mejorar su funcionalidad y disminuir las comorbilidades metabólicas. Desde julio del 2000 la Administración de Medicamentos y Alimentos de Estados Unidos de América (Food and Drug Administration, FDA) aprobó la indicación de hormona de crecimiento como opción terapéutica para el tratamiento del fallo de crecimiento en niños con síndrome de Prader-Willi. Actualmente este tratamiento constituye uno de los pilares en estos pacientes debido a que mejora calidad de vida, crecimiento, composición corporal, fuerza y tono musculares, agilidad y funcionalidad. ${ }^{4,5} \mathrm{No}$ hay un consenso sobre la edad ideal de inicio del tratamiento, sin embargo cada vez se inicia a edades más tempranas. También se ha documentado beneficio a dosis menores en adultos. ${ }^{4}$

No obstante, en el 2002 se reportaron casos de muerte de niños tratados con hormona de crecimiento, lo que por un tiempo se cuestionó la seguridad del medicamento. Tauber y sus colaboradores exploraron dicha asociación y en el 2008 publicaron la serie de casos más grande de pacientes pediátricos con síndrome de Prader-Willi fallecidos. Con la información de 64 pacientes (28 de ellos con tratamiento con hormona de crecimiento) describieron los siguientes resultados: 28 (44\%) de las muertes se atribuyeron a infecciones respiratorias; 11 (17\%) a insuficiencia respiratoria; 11 (17\%) a muerte súbita, 9 (14\%) a 
otras infecciones y $3(5 \%)$ por atragantamiento. $\mathrm{Al}$ realizar la comparación entre pacientes tratados o no con hormona de crecimiento (Cuadro 1) no encontraron diferencias estadísticamente significativas, con excepción de la edad (6.4 vs. 3.5 años, respectivamente $[p<0.001]$ ). Dentro del grupo tratado con hormona de crecimiento se observó que $75 \%$ de las muertes ocurrió durante los primeros 9 meses del tratamiento, pudiendo señalar una posible asociación de empeoramiento clínico por problemas respiratorios. Los autores, en un intento explicativo de causalidad, sugirieron que el tratamiento con hormona de crecimiento pudiera contribuir a hipertrofia adenoidea o amigdalina y empeorar la obstrucción de la vía aérea. Actualmente se recomienda una evaluación otorrinolaringológica y de trastornos respiratorios del sueño en los pacientes antes de iniciar tratamiento con hormona de crecimiento. ${ }^{1,6}$ Las contraindicaciones de para la hormona de crecimiento son obesidad grave, diabetes mellitus no controlada, apnea obstructiva del sueño grave no tratada, cáncer activo y psicosis. ${ }^{7,8}$

El diagnóstico de síndrome de Prader-Willi explica la mayoría de los datos clínicos del paciente en discusión. Sin embargo, vale la pena hacer un análisis más profundo con respecto a los posibles factores que explican el desenlace de este caso. El paciente en discusión tenía obesidad grave, estridor persistente desde el nacimiento y, claramente, un proceso infeccioso febril, tres factores que han sido consistentemente asociados con mortalidad en pacientes pediátricos con síndrome de Prader-Willi. ${ }^{1,9}$ Además de estos factores, el paciente era hijo de madre adolescente, con falta de apego al seguimiento y con un esquema de inmunizaciones incompleto. Con todos estos factores en contra el paciente llegó a nuestra institución y estuvo hospitalizado durante 10 días hasta su defunción. El evento final fue a su vez abrupto y resistente al tratamiento. De acuerdo con los datos identificados clínicamente (deterioro ventilatorio, palidez, hipotensión y sangrado por cánula) y por imagen (radiopacidad basal derecha con broncograma aéreo) pareciera que el deterioro se asoció con un evento agudo y catastrófico pulmonar.

Varios autores han descrito una mayor mortalidad global en pacientes con síndrome de Prader-Willi comparados con la población

Cuadro 1. Mortalidad en pacientes pediátricos con síndrome de Prader-Willi con y sin tratamiento con hormona de crecimiento

\begin{tabular}{|c|c|c|c|}
\hline Causa de muerte & $\begin{array}{l}\text { Con hormona de crecimiento } \\
\qquad(n=25)\end{array}$ & $\begin{array}{l}\text { Sin hormona de crecimiento } \\
\qquad(n=14)\end{array}$ & $p$ \\
\hline Causas respiratorias & $17(68 \%)$ & $7(50 \%)$ & NS \\
\hline Infección & $12(48 \%)$ & $6(43 \%)$ & \\
\hline Insuficiencia & $5(20 \%)$ & $1(7 \%)$ & \\
\hline Gastroenteritis & 0 & $2(14 \%)$ & NS \\
\hline Muerte súbita & $7(28 \%)$ & $4(29 \%)$ & NS \\
\hline Accidente & 0 & $1(7 \%)$ & NS \\
\hline Evento cardíaco & $1(4 \%)$ & 0 & NS \\
\hline Mediana de edad en años (rango) & $6.4(3-15.8)$ & $3.5(3-19)$ & $<0.0001$ \\
\hline Género $(\mathrm{m} / \mathrm{f})$ & $19 / 6$ & $8 / 6$ & NS \\
\hline Prevalencia de obesidad & $82 \%(19 / 23)$ & $62 \%(8 / 13)$ & NS \\
\hline Prevalencia de SAHOS & $32 \%(8 / 25)$ & $7 \%(1 / 14)$ & NS \\
\hline $\begin{array}{l}\text { Duración de tratamiento con hormona de creci- } \\
\text { miento en meses (rango) }\end{array}$ & $4(0.43-48)$ & - & \\
\hline
\end{tabular}


general 3 vs. $0.13 \%$ por año, principalmente por complicaciones de la obesidad (i.e. cor pulmonale, insuficiencia respiratoria hipercápnica, insuficiencia cardíaca, etcétera). ${ }^{9}$ Sin embargo, la morbilidad y mortalidad difieren de acuerdo con la edad. La mortalidad en niños con síndrome de Prader-Willi no es frecuente, habitualmente se presenta súbitamente y en asociación con infecciones respiratorias y fiebre. La mortalidad en adultos generalmente se asocia con causas circulatorias o respiratorias a su vez asociadas con obesidad. ${ }^{10}$

Los niños con síndrome de Prader-Willi padecen problemas respiratorios más frecuentemente y más graves que sus pares sanos. Esto obedece a múltiples factores pero parece explicarse principalmente por insuficiencia de los músculos respiratorios, alteraciones de la deglución, estrechez de la vía respiratoria superior, restricción de la ventilación, alteraciones centrales del control de la respiración e hipertensión pulmonar. ${ }^{11} \mathrm{Sin}$ embargo, el paciente contaba con asistencia mecánica de la ventilación por lo que estos factores parecieran no jugar un papel importante en el desenlace del paciente.

En un caso semejante al nuestro, Zellweger \& Schneider describieron un preescolar de 5 años de edad que cursó con somnolencia, anorexia y disnea durante 3 días y finalmente falleció. La autopsia documentó infiltración grasa del miocardio, edema pulmonar bilateral y neumonitis. ${ }^{12}$ Bray y sus colaboradores reportaron tromboembolia pulmonar masiva en una autopsia de un paciente de 3 años con síndrome de PraderWilli que murió hospitalizado. ${ }^{13}$ Stevenson y sus colegas reportaron 10 pacientes con síndrome de Prader-Willi con muerte no explicada o enfermedades críticas con presentaciones súbitas. Cinco casos menores de 2 años se caracterizaron por fallecer durante o cerca de un proceso infeccioso febril. En tres de cuatro casos de los que se realizó autopsia y se describieron las glándulas adrenales se documentó un peso muy por debajo del esperado para la longitud, dando plausibilidad a la insuficiencia adrenal. Un paciente cursó con una cardiomiopatía. Cinco de los 10 casos fallecieron durante el sueño. Con dicha información los autores comentan que los pacientes con síndrome de Prader-Willi pueden tener riesgo de enfermedades críticas desproporcionadas o muerte no explicable. ${ }^{14}$

Eiholzer publicó la información de 23 pacientes pediátricos con síndrome de Prader-Willi finados. Diecisiete de ellos (74\%) fallecieron por infecciones, 5 (22\%) por apnea del sueño o hipoventilación/aspiración y 1 (4\%) accidentalmente. El curso clínico de dichos eventos infecciosos fue abrupto y más corto de lo esperado; la muerte se presentó más bien súbita e inesperadamente. ${ }^{11}$ De Lind van Wijngaarden y su grupo reportaron hasta $60 \%$ de insuficiencia adrenal central en pacientes con síndrome de Prader-Willi, lo que presumiblemente condiciona una respuesta deficiente ante eventos de estrés y probablemente guarde relación con la elevada mortalidad ante procesos infecciosos. Los autores sugieren que se debe considerar el tratamiento con hidrocortisona durante procesos agudos de la enfermedad, a menos que se haya descartado insuficiencia adrenal. ${ }^{15}$

\section{COMENTARIO ANATOMOPATOLÓGICO}

El estudio post mortem se realizó en un niño obeso con peso de $27 \mathrm{~kg}$ y talla de $106 \mathrm{~cm}$ pero con manos y pies pequeños. El cerebro estaba disminuido de tamaño, con circunvoluciones delgadas, tortuosas y surcos amplios. Al corte se encontró leve hidrocefalia ex vacuo (Figura 1). Estos datos de atrofia son inespecíficos y son los que se han descrito en el síndrome de Prader-Willi. ${ }^{16}$ En los ganglios basales y el tálamo del lado izquierdo se encontró una zona de destrucción del parénquima con proliferación de gemistocitos y microglía espumosa perivas- 


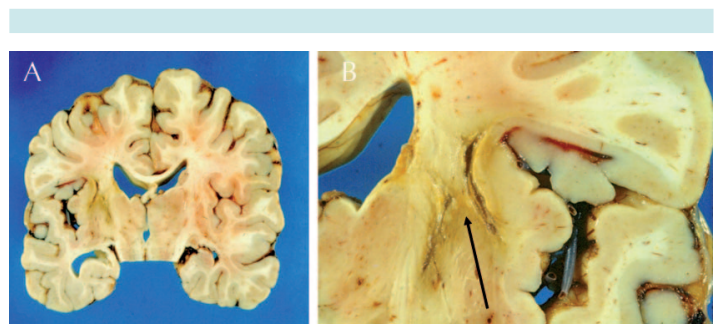

Figura 1. Corte coronal de cerebro. A) Atrofia y ligera dilatación ventricular (hidrocefalia ex vacuo). B) Acercamiento de núcleos basales del hemisferio izquierdo con destrucción lineal del tejido.

cular, que corresponden a un infarto antiguo seguramente relacionado con alguno de los episodios hipóxico isquémicos que presentó este niño (Figura 2). Las afecciones predominantes se encontraron en el aparato cardiovascular y en los pulmones. El corazón estaba aumentado de peso (230 g vs. $150 \mathrm{~g}$ ) con engrosamiento concéntrico del miocardio de ambas cavidades ventriculares. Las arterias pulmonares derecha e izquierda estaban parcialmente ocluidas por trombos de color amarillento, adheridos a la pared vascular y que se extendían desde la bifurcación del tronco hasta la entrada a nivel del hilio pulmonar. Ambos pulmones se encontraron aumentados de peso con la superficie pleural violácea y adherencias laxas. Al corte, el lóbulo

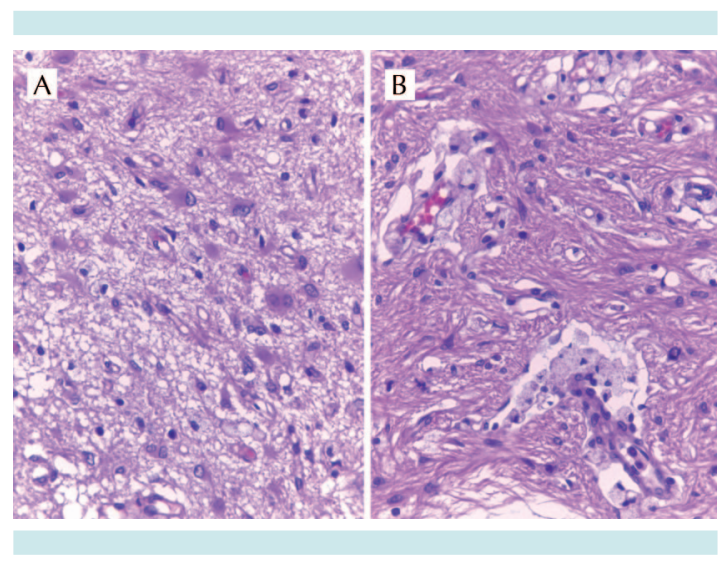

Figura 2. Infarto antiguo en núcleos basales izquierdos. A) Proliferación de astrocitos gemistocíticos. B) Microglía espumosa en espacio perivascular. inferior derecho presentaba una zona hemorrágica triangular de base pleural con destrucción del parénquima adyacente y en la cara interna una cavidad, de $2 \mathrm{~cm}$ de diámetro con bordes anfractuosos, abierta a la superficie pleural (Figura 3). El pulmón izquierdo tenía áreas consolidadas de consistencia firme alternando con zonas congestivas. Histológicamente, el parénquima pulmonar presentó necrosis hemorrágica en la cercanía de los vasos arteriales ocluidos y bronconeumonía abscedada en el lóbulo inferior derecho. Además se encontraron múltiples áreas de fibrosis pulmonar que corresponden a cicatrices de probables procesos neumónicos previos de isquemia. El hígado tenía aumento de tamaño, y esteatosis de gota gruesa afectando más de $40 \%$ de los hepatocitos, relacionado con el problema de obesidad (Figura 4). Los trombos presentaban grados de organización variable, algunos con recanalización (Figura 5).

La obesidad en adultos es un factor de riesgo reconocido para el desarrollo de tromboembolia pulmonar; sin embargo, esta complicación poca veces se ha documentado en niños. En este caso consideramos que la tromboembolia pulmonar masiva fue la responsable del cuadro clínico final

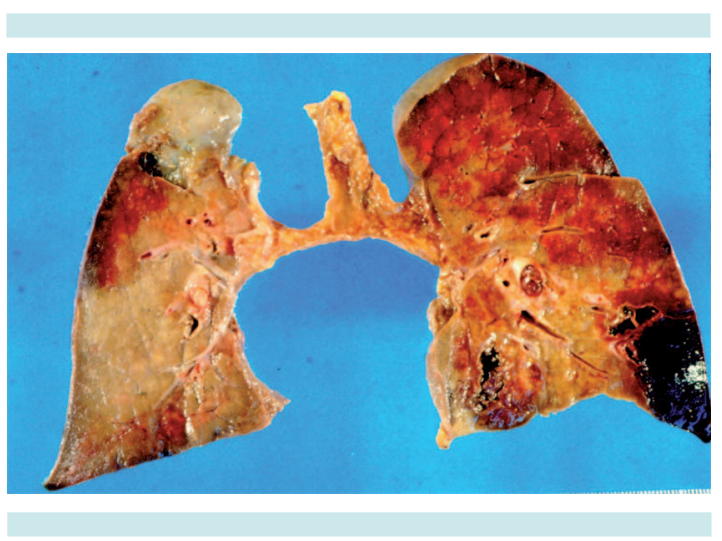

Figura 3. Corte sagital de ambos pulmones, vista posterior. En la base derecha se aprecian el infarto hemorrágico y un vaso ocluido (flecha). En el mismo lóbulo en la cara medial hay un absceso abierto a pleura. El pulmón izquierdo consolidado con infartos en lóbulo inferior. 


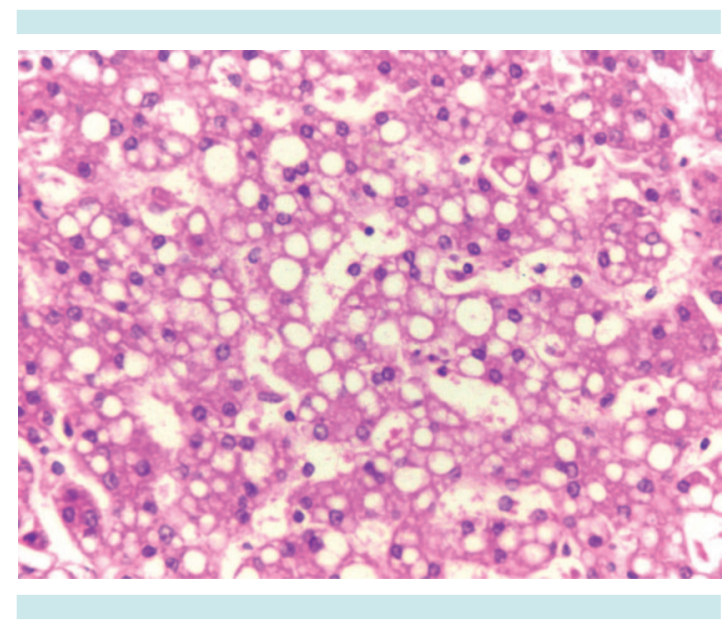

Figura 4. Hígado: esteatosis de gota gruesa (90\%).

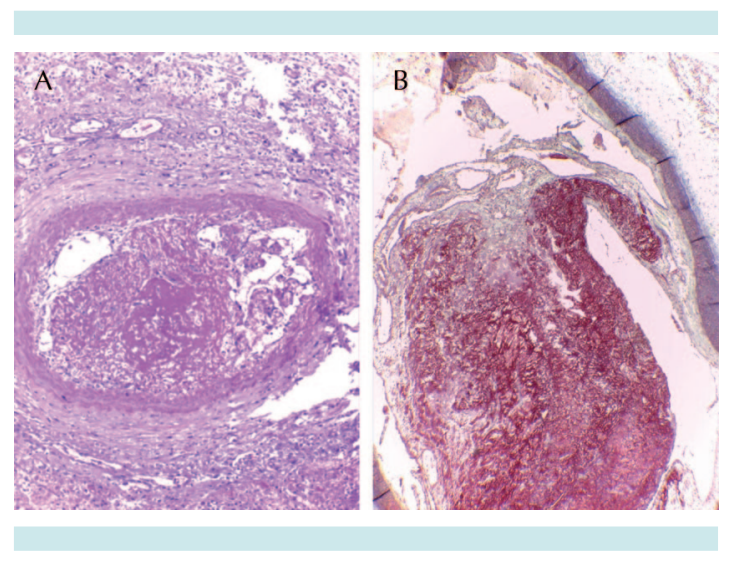

Figura 5. Arteria pulmonar. A) Tinción de hematoxilina y eosina: secciones de ramas de arteria pulmonar que muestran trombos en organización. B) Tinción de Masson: engrosamiento endotelial, adherencia al trombo y revascularización en la periferia.

y causante de la muerte. Es probable que esta complicación en niños obesos sea más frecuente de lo que se conoce. La tromboembolia pulmonar en niños ofrece mayor dificultad diagnóstica, pocas veces se piensa en ella y por lo tanto los métodos diagnósticos no se realizan en vida. En una revisión de autopsias del Departamento de Patología del Instituto Nacional de Pediatría se encontraron 22 casos de tromboembolia pulmonar fatal y solamente en 3 casos se hizo el diagnóstico en vida. ${ }^{17}$
En este caso no se encontró el origen del trombo. Suponemos que debe haber estado en las venas profundas de los miembros inferiores que no suelen explorarse en estudios post mortem convencionales. En el resto de los órganos se encontraron datos de septicemia con esplenitis aguda y choque con necrosis tubular y necrosis hepática multifocal hipóxico-isquémica. Las glándulas suprarrenales no mostraron alteraciones.

Diagnósticos anatómicos finales:

- $\quad$ Síndrome de Prader-Willi

Obesidad (27 kg vs. 18 kg)

Manos y pies pequeños

- Hipogonadismo

- Despoblación neuronal

- Atrofia cerebral, historia clínica de retraso mental

- Esofagitis crónica por reflujo

- Tromboembolias en arterias pulmonares derecha e izquierda recientes y organizadas.

- Infartos en lóbulos basales de ambos pulmones.

- Cardiomegalia global (230 g vs. 150 g) con hipertrofia biventricular.

- Esteatosis hepática.

- Bronconeumonía bilateral con absceso en lóbulo basal derecho abierto a cavidad pleural.

- Neumonía organizada.

- Infarto antiguo en núcleos basales izquierdos.

- Septicemia (esplenitis séptica).

- Datos anatómicos de choque:

- Daño alveolar difuso

- Necrosis hepática hipóxico isquémica

- Necrosis tubular aguda 


\section{COMENTARIO CLÍNICO}

La obesidad, un proceso infeccioso, la estancia hospitalaria prolongada en unidad de cuidados críticos con apoyo mecánico a la ventilación, dispositivos invasivos y poca o nula movilidad son factores de riesgo descritos para tromboembolia pulmonar en población adulta. ${ }^{18} \mathrm{Sin}$ embargo, en población pediátrica la tromboembolia pulmonar no ocurre frecuentemente y no se realizan intervenciones preventivas de manera rutinaria. Publicaciones recientes afirman que los diagnósticos de trombosis venosa profunda y tromboembolia pulmonar son subestimados en estos grupos etarios. ${ }^{19-21}$ Una revisión sistemática reciente estimó una prevalencia de trombosis venosa profunda de 9.7/10 000 hospitalizaciones pediátricas. Tras analizar la información de 761 pacientes pediátricos hospitalizados, que cursaron con algún evento de trombosis venosa profunda o tromboembolia pulmonar, se identificaron los siguientes factores de riesgo: catéter venoso central, proceso infeccioso, cirugía, malignidad, traumatismo, cardiopatía, síndrome nefrótico, obesidad. El $74 \%$ de las trombosis venosas profundas ocurrieron en miembros inferiores, mientras que $26 \%$ en miembros superiores. En 124 (16\%) pacientes ocurrió tromboembolia pulmonar, con un desenlace fatal en $12(10 \%)$ de ellos. ${ }^{22}$

Otro aspecto que nuestro paciente presentó clínicamente y en el estudio post mortem fueron las infecciones de las vías respiratorias. Como se comentó previamente estos procesos constituyen la principal causa de muerte en niños con síndrome de Prader-Willi. ${ }^{1}$ A pesar de este dato, dicha susceptibilidad no ha sido explorada desde el punto de vista inmunológico. Los factores anatómicos (patrón restrictivo, obstrucción y reducción del calibre de la vía respiratoria) y funcionales (hipotonía muscular, hiperfagia, trastorno central del control de la respiración) pueden explicar hasta cierto grado tal vulnerabilidad..$^{1,13-15}$ Sin embargo, otro fenómeno frecuentemente descrito en la literatura es que ni la evolución de los procesos infecciosos ni los datos clínicos de los pacientes con síndrome de Prader-Willi suelen correlacionar con la gravedad de la enfermedad. ${ }^{14}$ Curiosamente, Grzmil y sus colaboradores desarrollaron un modelo murino genéticamente modificado, sin expresión del gen que traduce para proteína tipo 3 con dominio tipo NIPA (NIPA-like domain containing protein 3, Npal3 -/-); esta proteína está indirectamente afectada en los pacientes con síndrome de Prader-Willi por la translocación de los genes NIPA con los que interactúa. Los autores describieron disminución cuantitativa de células asesinas naturales, así de concentraciones de $\lg$, IgG2, IgA e incremento en la síntesis de IgE, evidenciando un compromiso en la función del sistema inmunológico de este modelo murino. ${ }^{23}$ Dichos hallazgos ofrecen un campo nuevo de investigación clínica y posiblemente otras opciones terapéuticas en pacientes con síndrome de Prader-Willi.

Considerando que, con excepción del diagnóstico de base y la posibilidad de insuficiencia adrenal central, los otros factores de riesgo presentes son comunes a muchos otros pacientes que se hospitalizan (obesidad, ingreso a cuidados críticos, inmovilización prolongada, uso de catéteres intravenosos, procesos infecciosos) la trombosis venosa profunda y la tromboembolia pulmonar en población pediátrica probablemente sean más frecuentes de lo que consideramos y seguramente ofrecen una oportunidad de estudiar y proponer estrategias de prevención o tratamiento.

\section{REFERENCIAS}

1. Tauber M, Diene G, Molinas C, He'bert M. 2008. Review of 64 cases of death in children with Prader-Willi syndrome (SPW). Am J Med Genet Part A 146A:881-887.

2. Butler MG. 1990. Prader-Willi syndrome: current understanding of cause and diagnosis. Am J Med Genet 35:319-332. 
Padrón-Martínez M et al. Síndrome Prader Willi y tromboembolia pulmonar

3. Hertz G, Cataletto M, Feinsilver SH, Angulo M. 1993. Sleep and breathing patterns in patients with Prader Willi syndrome (SPW): effects of age and gender. Sleep 16:366-371.

4. Stephen F Kemp, J Paul Frindik. Emerging options in growth hormone therapy: an update. Drug Des Devel Ther 2011;5:411-419.

5. Donald G. Goranson, Jr. Growth Hormone and Prader Willy Syndrome. Second Edition. A reference for families and care providers. Prader-Willy Syndrome Association 2011. pp. 7-8.

6. Dong Kyu Jin. Systematic review of the clinical and genetic aspects of Prader-Willi syndrome. Korean J Pediatr 2011;54(2):55-63.

7. Deal CL, Tony M, Höybye C, Allen DB, Tauber M, Christiansen JS, et al. Growth Hormone Research Society workshop summary: consensus guidelines for recombinant human growth. 98(6):E1072-E1087.

8. Yea Ji Kim, Chong Kun Cheon. Prader-Willi syndrome: a single center's experience in Korea. Korean J Pediatr 2014;57(7):310-316.

9. Whittington JE, Holland AJ, Webb T, Butler J, Clarke D, Boer $\mathrm{H}$ : Population prevalence and estimated birth incidence and mortality rate for people with Prader-Willi syndrome in one UK Health Region. J Med Genet 2001;38:792-798.

10. Einfeld SL., Kavanagh SJ, Smith A, Evans EJ, Tonge BJ, Taffe J. Mortality in Prader-Willi Syndrome. Am J Ment Retard. 2006;111(3):193-198.

11. Eiholzer U. Deaths in Children with Prader-Willi Syndrome. Horm Res 2005;63:33-39.

12. Zellweger $\mathrm{H}$, Schneider HJ. Syndrome of hypotoniahypomentiahypogonadism-obesity ( $\mathrm{HHHO}$ ) or Prader-Willi syndrome. Amer J Dis Child 1968;115:588-598.

13. Bray GA, Dahms WT, Swerdloff RS, Fiser RH, Atkinson RL, Carrel RE. The Prader-Willi syndrome: a study of 40 patients and a review of the literature. Medicine (Baltimore) 1983;62(2):59-80.

14. Stevenson DA, Anaya TM, Clayton-Smith J, Hall BD, Van Allen MI, Zori RT \& Clericuzio CL. Unexpected death and critical illness in Prader-Willi syndrome: report of ten individuals. American Journal of Medical Genetics Part A 2004;24(2):158-164.

15. de Lind van Wijngaarden RFA, Otten BJ, Festen DAM, Joosten KFM, de Jong FH, Sweep FCGJ, Hokken-Koelega ACS. Central Adrenal Insufficiency in SPW patients. J Clin Endocrinol Metab 2008;93(5):1649-1654.

16. Schrander-Stumpel CT, Curfs LM, Sastrowijoto P, Cassidy SB, Schrander JJ, Fryns JP. Prader-Willi syndrome: causes of death in an international series of 27 cases. Am J Med Genet A 2004;124A:333-8.

17. Ridaura-Sanz C. Ovando-Fonseca JE, López-Corella E. Tromboembolia pulmonar fatal en la edad pediátrica. Memorias de la CII Reunión Reglamentaria AIP Junio. 2006. pp. 75-85.

18. Heit JA, Silversten MD, Mohr DN, Petterson TM, O’Fallon WM, Melton LJ. Risk Factors for Deep Vein Thrombosis and Pulmonary Embolism. A population-based Case-control study. Arch Intern Med 2000;160:809-815.

19. Sandoval JA, Sheehan MP, Stonerock CE, Shafique S, Rescorla FJ, Dalsing MC. Incidence, risk factors, and treatment patterns for deep venous thrombosis in hospitalized children: an increasing population at risk. J Vasc Surg 2008;47:837-843.

20. Biss TT, Brandao LR, Kahr WH, Chan AK, Williams S. Clinical features and outcome of pulmonary embolism in children. Br J Haematol 2008;142:808-818.

21. Vu LT, Nobuhara KK, Lee H, Farmer DL. Determination of risk factors for deep venous thrombosis in hospitalized children. J Pediatr Surg 2008;43:1095-1099.

22. Kim SJ, Sabharwal S. Risk factors for venous thromboembolism in hospitalized children and adolescents: a systemic review and pooled analysis. J Pediatr Orthop B 2014;23(4):389-393.

23. Grzmil P1, Konietzko J, Boehm D, Hölter SM, Aguilar-Pimentel A, Javaheri A, et al. Targeted disruption of the mouse Npal3 gene leads to deficits in behavior, increased IgE levels, and impaired lung function. Cytogenet Genome Res 2009;125(3):186-200. doi: 10.1159/000230003. Epub 2009 Sep 4. 